\title{
USO DE LA PUNCIÓN LUMBAR EN LA EVALUACIÓN DE SEPSIS NEONATAL TARDÍA EN RECIÉN NACIDOS DE BAJO PESO
}

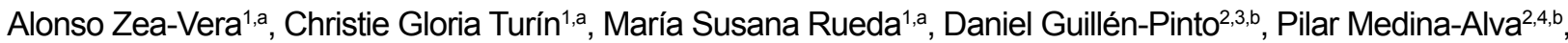

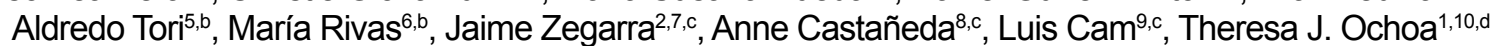

\section{RESUMEN} \\ El objetivo del estudio fue analizar el uso de la punción lumbar $(\mathrm{PL})$ en las sospechas de sepsis neonatal tardía. Se utilizó \\ una cohorte de 414 neonatos con peso al nacer $<2000 \mathrm{~g}$ en tres hospitales de Lima. Se realizó la PL en $45 / 214(21,0 \%)$ \\ sospechas de sepsis y en $13 / 48(27,1 \%)$ sepsis confirmadas por hemocultivo. Se diagnosticó meningitis en 8/214 $(3,7 \%)$ \\ sospechas y en $8 / 45(17,5 \%)$ episodios en los que se realizó la PL. El tiempo de tratamiento de los episodios sin PL \\ fue similar a los episodios de sepsis con meningitis descartada y menor a los episodios de meningitis. El uso de la PL \\ es bajo, lo que puede resultar en meningitis no diagnosticadas y tratadas inadecuadamente. Es necesario reforzar la \\ importancia de la PL en la evaluación de sepsis neonatal.
}

Palabras clave: Meningitis, Punción Espinal, Sepsis, Recién Nacido (fuente: DeCS BIREME).

\section{USE OF LUMBAR PUNCTURE IN THE EVALUATION OF LATE-ONSET SEPSIS IN LOW BIRTH WEIGHT NEONATES}

\begin{abstract}
The objective of this study was to analyze the use of lumbar punctures (LP) in the evaluation of late-onset neonatal sepsis. It is recommended to perform an LP as part of the evaluation of late-onset sepsis. We used a cohort of 414 newborns with a birth weight $<2000 \mathrm{~g}$ in three hospitals in Lima. A LP was performed in 45/214 $(21.0 \%)$ of sepsis evaluations and in $13 / 48(27.1 \%)$ of culture-proven sepsis. Meningitis was diagnosed in $8 / 214(3.7 \%)$ of the episodes and $8 / 45(17.5 \%)$ of the evaluations that included an LP. The duration of treatment of the sepsis episodes without a LP and the episodes with a negative LP was similar, and shorter than the episodes with a positive LP. The use of LP in the evaluation of late-onset sepsis is low and can result in undiagnosed and undertreated meningitis. The use of LP in the evaluation of neonatal sepsis must be encouraged in the neonatal units.
\end{abstract}

Keywords: Meningitis, Spinal Puncture, Infant, Newborn (source: MeSH NLM).

\section{INTRODUCCIÓN}

La meningitis neonatal está asociada a una alta mortalidad y discapacidad a largo plazo ${ }^{(1,2)}$. Se presenta en $0,25-$ 0,44 por 1000 nacidos vivos ${ }^{(3,4)}$ y en $6,5-14$ por 1000 neonatos de muy bajo peso al nacer $(<1500 \mathrm{~g})^{(2,5)}$. En un tercio de los casos -y dos tercios de las meningitis por
Cándida -no se logra aislar un germen en la sangre ${ }^{(2,6,7)}$. La meningitis es más frecuente en episodios de sepsis neonatal tardía que en sepsis temprana ${ }^{(8,9)}$. De los recién nacidos evaluados por sepsis tardía, 1,3-3,5\% son diagnosticados de meningitis ${ }^{(10,11)}$.

La punción lumbar $(\mathrm{PL})$ sirve para guiar la duración de la terapia antibiótica. El tiempo recomendado para un

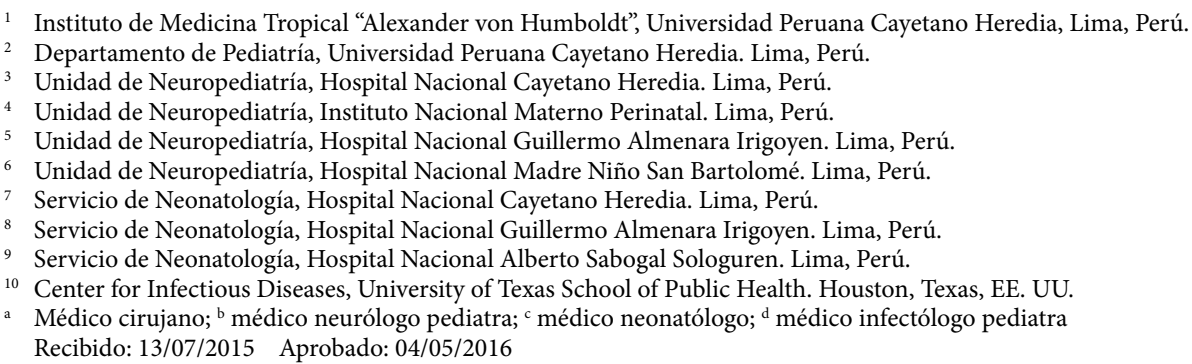


episodio de sepsis sin meningitis es de 7-10 días, en el caso de encontrarse una meningitis el tiempo se prolonga a 14 días para gérmenes Gram-positivos, y 21 días para Gram-negativos ${ }^{(12)}$. Un tratamiento inadecuado podría aumentar la mortalidad y morbilidad asociada con esta enfermedad. Actualmente, se recomienda que la PL se realice como parte de la evaluación de sepsis tardía ${ }^{(13)}$.

Sin embargo, el uso de la $\mathrm{PL}$ en neonatos es controversial ya que no existen guías clínicas aceptadas universalmente $\mathrm{y}$, por lo tanto, su práctica varía entre diferentes hospitales ${ }^{(2)}$. Una encuesta a neonatólogos australianos encontró que solo el $51 \%$ realizan una $\mathrm{PL}$ de rutina ante la sospecha de sepsis tardía ${ }^{(14)}$. El objetivo de este estudio es reportar el uso de la PL en la evaluación de sospechas de sepsis tardías en tres unidades de cuidados intensivos neonatales de Lima.

\section{EL ESTUDIO}

Este estudio se realizó a través de un análisis secundario de la base de datos del ensayo clínico "Lactoferrina para prevención de sepsis neonatal" (NEOLACTO), cuyo objetivo es evaluar la suplementación oral con lactoferrina para la prevención de sepsis tardía en neonatos (identificador de ClinicalTrials.gov: NCT01525316). Se incluyeron neonatos con un peso al nacer de 500-2000 g internados en la unidad de cuidados intensivos o intermedios del Hospital Nacional Guillermo Almenara Irigoyen, Hospital Nacional Cayetano Heredia y Hospital Nacional Alberto Sabogal Sologuren. Los pacientes fueron enrolados antes de las $72 \mathrm{~h}$ de vida. Se excluyeron a los neonatos con problemas gastrointestinales que impidan la vía oral, condiciones subyacentes que afecten profundamente el crecimiento y desarrollo, historia familiar de alergia a la proteína de la leche de vaca y dificultades para completar un seguimiento de 2 años.

Cada sospecha de sepsis tardía fue registrada, incluyendo día de vida, exámenes de laboratorio tomados, resultados de cultivos y terapia antibiótica. Se definió como sospecha de sepsis tardía cualquier episodio caracterizado por un cambio clínico o de laboratorio sugerente de sepsis neonatal según los criterios de Haque (15) luego de las 72 h de vida. Cada episodio fue discutido por el médico tratante y la investigadora principal del ensayo (OTJ). Usando una versión modificada de los criterios de Haque (15) los episodios fueron clasificados en una de cuatro categorías: sepsis confirmada, sepsis probable, sepsis posible o sepsis descartada.

En este estudio se incluyeron a todos los pacientes con al menos una sospecha de sepsis. Por cada sospecha se tomaron los siguientes datos: clasificación del episodio, realización de al menos una $\mathrm{PL}$, número de días luego del inicio del episodio en el que se realizó la $\mathrm{PL}$, diagnóstico de meningitis neonatal, germen aislado en sangre, germen aislado en líquido cefalorraquídeo (LCR) y duración de la terapia antibiótica del episodio. La meningitis neonatal se definió como cualquier sospecha de sepsis con un cultivo de LCR positivo o pleocitosis en el LCR en el contexto de una sepsis confirmada o probable. Para este estudio se consideraron como infecciones tardías (sepsis o meningitis) a aquellas que se presentan luego de las $72 \mathrm{~h}$ de vida.

La unidad de análisis de este estudio fueron los episodios de sospechas de sepsis, por lo tanto se incluyó más de un episodio por paciente. Se analizó la frecuencia de $\mathrm{PL}$, tiempo transcurrido para realizar la $\mathrm{PL}$ y diagnóstico de meningitis según tipo de sospecha de sepsis y germen causante. Para la descripción de las variables cualitativas se usaron proporciones. Para la descripción de las variables cuantitativas se usó la mediana y el rango intercuartil (RIQ) o media y desviación estándar, dependiendo de la distribución de la variable. Para determinar la normalidad se usó el análisis gráfico de las variables. Además, se compararon las duraciones de terapia antibiótica de acuerdo a realización de PL y diagnóstico de meningitis. La prueba de Fisher se usó para comparar las variables cualitativas y la prueba $U$ de Mann-Whitney o prueba t para comparar las variables continuas, dependiendo de su distribución. El análisis se hizo en el programa Stata versión 11.

El ensayo clínico NEOLACTO ha sido aprobado por el comité de ética de la Universidad Peruana Cayetano Heredia, la Universidad de Texas en Houston y los comités de cada uno de los hospitales participantes. Todos los datos de los pacientes se mantuvieron de forma confidencial almacenados en el servidor de la Universidad Peruana Cayetano Heredia con acceso con clave solo para los investigadores.

\section{HALLAZGOS}

Se enrolaron 414 neonatos, de estos, se excluyeron 273 debido a que no tuvieron sospechas de sepsis durante la hospitalización, dejando un total de 141 pacientes para nuestro análisis. El número máximo de sospechas por paciente fue 7 . La edad gestacional promedio de los neonatos con sospechas fue 29,8 $( \pm 2,9)$ semanas y el peso al nacer promedio fue 1223,3 $( \pm 344,1) \mathrm{g}$. Comparado a la cohorte total, este subgrupo tuvo un menor peso al nacer, menor edad gestacional, mayor score de Apgar a los 5 min y mayor tiempo de hospitalización (Tabla 1).

Se registraron 214 sospechas de sepsis tardía; 48 $(22,4 \%)$ fueron clasificadas como confirmadas; 46 
Tabla 1. Características de la cohorte

\begin{tabular}{lrr}
\hline & $\begin{array}{c}\text { Neonatos con sospecha de sepsis } \\
(\mathbf{N = 1 4 1 )}\end{array}$ & $\begin{array}{c}\text { Cohorte total } \\
(\mathbf{N}=\mathbf{4 1 4})\end{array}$ \\
\hline Peso al nacer en $\mathrm{g}(\mathrm{m}, \mathrm{de})^{*}$ & $1223,3(344,1)$ & $1380,1(362,0)$ \\
Edad gestacional $(\mathrm{m}, \mathrm{de})^{*}$ & $29,8(2,9)$ & $30,8(3,0)$ \\
Sexo masculino $(\mathrm{n}, \%)$ & $84(59,6 \%)$ & $230(55,6 \%)$ \\
Apgar a los 5 min (m, de) & $8,2(1,3)$ & $6,9(2,0)$ \\
Cesárea $(\mathrm{n}, \%)$ & $116(82,3 \%)$ & $332(80,4 \%)$ \\
Preeclampsia $(\mathrm{n}, \%)$ & $36(25,5 \%)$ & $122(29,5 \%)$ \\
Esteroides antenatales $(\mathrm{n}, \%)$ & $94(66,7 \%)$ & $293(70,7 \%)$ \\
Infección periparto $(\mathrm{n}, \%)$ & $40(28,4 \%)$ & $104(25,1 \%)$ \\
Días de hospitalización $(\mathrm{m}, \mathrm{de})^{*}$ & $47,3(28,2)$ & $32,4(24,1)$ \\
\hline
\end{tabular}

m: media; de: desviación estándar; n: sujetos con la característica, \% porcentaje ${ }^{*} p<0,05$

$(21,5 \%)$ como probables; 16 (7,5\%) como posibles, y 94 $(43,9 \%)$ como descartadas. De las sepsis confirmadas $24(50,0 \%)$ fueron por Gram-positivos; 18 (37,5\%) por Gram-negativos, y 6 (12,5\%) por hongos. En tres episodios se aislaron dos patógenos (dos Gramnegativos en dos de ellos, y un Gram-negativo y un Gram-positivo en otro). Estos tres episodios fueron clasificados, para fines de este reporte, como sepsis por Gram-negativo debido a que este tipo de germen requiere mayor tiempo de tratamiento.

Se realizó al menos una $\mathrm{PL}$ en 45/214 (20,9\%) sospechas y en $13 / 46(27,1 \%)$ de las sepsis confirmadas (Tabla 2). La mediana (RIQ) del tiempo entre el inicio de la sospecha y la PL fue de 1 (0-2) día. El uso de la PL varía en cada hospital (Tabla 2). En el hospital A no se encontró una asociación entre la frecuencia de PL y la clasificación de la sospecha $(p=0,85)$. En el hospital $C$ se realizó la $\mathrm{PL}$ con mayor frecuencia en sepsis confirmadas y probables $(p<0,01)$. El hospital B solo realizó una PL en un caso de sepsis confirmada.

Se diagnosticó meningitis en $8 / 214(3,7 \%)$ sospechas, que representa el $17,8 \%(8 / 45)$ de las sospechas en donde se realizó una PL, y el 6,6\% (8/121) de todos los episodios de sepsis clínica (confirmada, probable y posible). Cinco casos de meningitis fueron diagnosticados en pacientes con sepsis probable, es decir sin hemocultivo positivo (Tabla 3). En tres se identificó el germen causante (1 Gram-negativo, infección mixta de Enterobacter sp. y E. coli; 1 Grampositivo, Enterococcus sp., y 1 hongo, Candida sp.). El Gram-positivo fue aislado en sangre y en LCR; el Gramnegativo se aisló solo en sangre junto con un examen de LCR anormal, y el hongo solo se aisló en LCR.

La mediana (RIQ) de días de antibióticos en los casos de meningitis fue 21,5 (16,5-23); en los episodios de sepsis sin meningitis fue $15(10-19)$ y en los episodios de sepsis sin punción lumbar fue 14 (11-19) (Tabla 4). Los episodios con meningitis recibieron una mayor duración de tratamiento que aquellos en los que se descartó la meningitis $(p=0,04)$ y aquellos en los que no se realizó una $\mathrm{PL}(p<0,1)$. No se encontró diferencia en la duración de la terapia entre los episodios en los que no se realizó una PL y los episodios en los que se descartó una meningitis $(p=0,69)$.

\section{DISCUSIÓN}

Encontramos que solo el $21,0 \%$ de las sospechas de sepsis -y el 2,3\% en uno de los hospitales- son evaluadas con una PL. En un reporte similar de 15 hospitales de Estados Unidos se hizo una PL en la mitad de las sospechas; la frecuencia en cada hospital varió de 22$85 \%{ }^{(2)}$. En este estudio, de los pacientes con sepsis confirmada, solo $27,1 \%$ tuvieron una $\mathrm{PL}$, comparado con $60,0 \%$ reportado en otras cohortes ${ }^{(2,13)}$.

Tabla 2. Frecuencia de punción lumbar según tipo de sospecha de sepsis y hospital

\begin{tabular}{lrrrr}
\hline & Hospital A & \multicolumn{1}{c}{ Hospital B } & \multicolumn{1}{c}{ Hospital C } & \multicolumn{1}{c}{ Total } \\
\hline Confirmada (n/N,\%) & $4 / 13(30,8 \%)$ & $1 / 4(25,0 \%)$ & $8 / 31(25,8 \%)$ & $13 / 48(27,1 \%)$ \\
Probable (n/N,\%) & $5 / 11(45,2 \%)$ & $0 / 12(0,0 \%)$ & $11 / 34(32,4 \%)$ & $16 / 57(28,1 \%)$ \\
Posible (n/N,\%) & $1 / 3(33,3 \%)$ & $0 / 7(0,0 \%)$ & $1 / 6(16,7 \%)$ & $2 / 16(12,5 \%)$ \\
Descartada (n/N, \%) & $14 / 44(31,8 \%)$ & $0 / 20(0,0 \%$ & $0 / 29(0,0 \%)$ & $14 / 93(15,1 \%)$ \\
Total de sospechas (n/N, \%) & $24 / 71(33,8 \%)$ & $1 / 43(2,3 \%)$ & $20 / 100(20,0 \%)$ & $45 / 214(21,0 \%)$ \\
\hline
\end{tabular}

$\mathrm{n}$ : sujetos con la característica; $\mathrm{N}$ : total en el grupo; \%: porcentaje 
Tabla 3. Frecuencia de meningitis por tipo de sospecha de sepsis

\begin{tabular}{lrr}
\hline & $\begin{array}{c}\text { Episodios } \\
\text { con punción } \\
\text { lumbar }\end{array}$ & \multicolumn{1}{c}{$\begin{array}{l}\text { Todos los } \\
\text { episodios }\end{array}$} \\
\hline Confirmada $(\mathrm{n} / \mathrm{N}, \%)$ & $3 / 13(23,1 \%)$ & $3 / 48(6,3 \%)$ \\
Probable $(\mathrm{n} / \mathrm{N}, \%)$ & $5 / 16(31,3 \%)$ & $5 / 57(8,8 \%)$ \\
Posible $(\mathrm{n} / \mathrm{N}, \%)$ & $0 / 2(0,0 \%)$ & $0 / 16(0,0 \%)$ \\
\hline Descartada $(\mathrm{n} / \mathrm{N}, \%)$ & $0 / 14(0,0 \%)$ & $0 / 93(0,0 \%)$ \\
\hline Total de sospechas $(\mathrm{n}, \mathrm{N}, \%)$ & $8 / 45(17,8 \%)$ & $8 / 214(3,7 \%)$ \\
\hline
\end{tabular}

$\mathrm{n}$ : sujetos con la característica; $\mathrm{N}$ : total en el grupo; \%: porcentaje

En dos de los tres hospitales evaluados el uso de la PL estuvo asociado a la clasificación final del episodio. Esto sugiere que se realizó la $\mathrm{PL}$ principalmente en aquellos neonatos con "mayor riesgo" de acuerdo a sus características clínicas y de laboratorio. Debido a la alta frecuencia de meningitis en los casos en que se realizó una PL $(17,8 \%)$ es probable que algunos casos se hayan perdido por falta de una PL.

Muchos estudios han buscado predictores clínicos de meningitis neonatal sin éxito ${ }^{(4,6,16)}$. El uso de la PL en neonatos ha sido evaluada en tres contextos: sepsis neonatal temprana, sepsis neonatal tardía y neonato febril $\sin$ foco en la emergencia. En sepsis neonatal temprana la única situación en la que es seguro obviar la PL es en casos de neonatos asintomáticos siendo evaluados únicamente por factores de riesgo materno ${ }^{(17)}$. En sepsis neonatal tardía algunos autores recomiendan realizar una punción lumbar únicamente en neonatos con hemocultivos positivos. Sin embargo, hasta un tercio de los casos de meningitis neonatal pueden presentarse con hemocultivos negativos ${ }^{(2)}$. Stoll et al. encontraron que a pesar que el uso de la PL varía entre hospitales, la tasa de positividad es similar entre ellos. Esto indica que la diferencia en la práctica no responde a un mejor "olfato" clínico ${ }^{(2)}$. Finalmente, en los neonatos febriles sin foco, evaluados en emergencia, no ha sido posible encontrar marcadores que permitan diferenciar una población con bajo riesgo de meningitis, por ello está recomendado realizar una PL a todo neonato febril sin foco ${ }^{(18)}$.
Se han reportado eventos adversos como hipoxemia severa y deterioro clínico con la PL en neonatos ${ }^{(19)} \mathrm{y}$, en ocasiones, la PL es postergada por considerar que el paciente está muy enfermo para tolerarla. Sin embargo, en una cohorte grande, la PL no se asoció a mayor mortalidad. Por lo contrario, la meningitis aumentó significativamente la mortalidad, el requerimiento de ventilación mecánica y la duración de hospitalización ${ }^{(2)}$. A largo plazo, la meningitis neonatal puede resultar en parálisis cerebral, déficit auditivo e hidrocefalia. El riesgo de no diagnosticar una meningitis es mayor al riesgo de realizar la PL.

Las sospechas de sepsis sin PL recibieron el mismo tiempo de tratamiento que episodios de sepsis con meningitis descartada. Esto puede resultar en una terapia subóptima en aquellos casos en los que no se diagnosticó meningitis por falta de una PL. Una posible solución a este problema es tratar a los episodios sin PL como si tuvieran meningitis. Sin embargo, el uso excesivo de antibióticos podría resultar en el aumento de resistencia antibiótica, infecciones fúngicas y mortalidad (20-22).

Las fortalezas de este estudio están en la recolección prospectiva de datos y la inclusión de tres hospitales diferentes. Una limitación es que solo hemos incluido a los pacientes enrolados en el ensayo NEOLACTO, perdiendo algunos casos de sospechas de sepsis. Estos pacientes tienen un mayor riesgo de meningitis y sepsis que la población general de las unidades neonatales, debido a su menor peso al nacer. Es probable que la mayoría de sospechas de sepsis se hayan encontrado en el grupo incluido. Otra limitación es el efecto de la intervención (lactoferrina) en la frecuencia de sepsis neonatal y meningitis. Sin embargo, al ser un estudio ciego, la intervención no debería afectar la decisión de usar una PL en la evaluación de los episodios.

En conclusión, el uso de la PL como parte de la evaluación de sepsis neonatal tardía es bajo y muy heterogéneo en los hospitales evaluados. En los casos de sepsis en los que no se realizó una PL el episodio es tratado como una sepsis con meningitis descartada. Esto puede resultar en meningitis no diagnosticadas y tratadas deficientemente.

Tabla 4. Duración de terapia antibiótica en días según tipo de sospecha, presencia de punción lumbar y meningitis

\begin{tabular}{|c|c|c|c|}
\hline & \multirow{2}{*}{ Sin punción lumbar } & \multicolumn{2}{|c|}{ Con punción lumbar } \\
\hline & & Sin meningitis & Con meningitis \\
\hline Confirmada (p50, RIQ) & $15(13-20)$ & $14.5(10-23)$ & $23(13-35)$ \\
\hline Fúngica (p50, RIQ) & $19(12-31)$ & - & $13^{*}$ \\
\hline Gram-negativo (p50, RIQ) & $16(14-24)$ & $19(11-27)$ & $23^{*}$ \\
\hline Gram-positivo (p50, RIQ) & $15(13-19)$ & $14.5(10-23)$ & $35^{*}$ \\
\hline Probable (p50, RIQ) & $12(9-15)$ & $15(9-18)$ & $21(18-22)$ \\
\hline Posible (p50, RIQ) & $11(11-17)$ & $13(11-15)$ & - \\
\hline Descartada (p50, RIQ) & $8(4-12)$ & $6,5(3-9)$ & - \\
\hline
\end{tabular}

*no se presenta el rango intercuartil porque solo hay una observación.

p50: mediana; RIQ: rango intercuartil. 
Por lo tanto, se recomienda enfatizar la importancia de la realización de la PL en la evaluación de sospechas de sepsis en las unidades neonatales y en los programas de entrenamiento de futuros neonatólogos. Esta práctica deberá evaluarse periódicamente e implementarse procedimientos que disminuyan las barreras a la realización de esta prueba diagnóstica.

Agradecimientos: agradecemos a Sicilia Bellomo, María Luz Rospigliosi, Geraldine Borda, Verónica Webb, Raul Llanos, Oscar Chumbes, Liliana Cuba, Carmen Chincaro, Ana Lino y Augusto Cama por su apoyo en el enrolamiento y seguimiento de los pacientes. Además, a Marlene Caffo, Amelia Bautista, Rosa Atencio, Rosa Grados, Brígida Mejía, Nelly Valverde, Nancy Cabrera, Clarisa Diaz, Giovanna García, Martha Torres, Elizabeth Rojas, Virginia Loo, Rosa Quijano, Luisa Osorio,
Carmen Huaranga, Amelia Escalante, María Marres, Lourdes Tucto, Rocío Lucas, Cristina Suarez, Mayela Huayanay y Nadia Rojas por su apoyo en el seguimiento de los pacientes.

Contribuciones de autoría: AZ-V, CGT, MSR y TJO participaron en la concepción y diseño del artículo y la redacción inicial del manuscrito. DG-P, PM-A, AT, MR, JZ, AC y LC participaron en el enrolamiento de pacientes y recolección de resultados. TJO obtuvo el financiamiento para el proyecto. AZ-V realizó el análisis e interpretación de datos. Todos los autores revisaron y aprobaron la versión final del manuscrito.

Fuentes de financiamiento: National Institute of Child Health \& Human Development (grant: R01HD067694), USA

Conflictos de interés: los autores de este manuscrito declaran no tener ninguna relación económica o institucional relevante a los resultados de este estudio.

\section{REFERENCIAS BIBLIOGRÁFICAS}

1. Bedford $\mathrm{H}$, de Louvois J, Halket $S$, Peckham C, Hurley R, Harvey D. Meningitis in infancy in England and Wales: follow up at age 5 years. BMJ. 2001;323(7312):533-6. doi: http:// dx.doi.org/10.1136/bmj.323.7312.533.

2. Stoll BJ, Hansen N, Fanaroff AA, Wright LL, Carlo WA, Ehrenkranz RA, et al. To tap or not to tap: high likelihood of meningitis without sepsis among very low birth weight infants. Pediatrics. 2004;113(5):1181-6.

3. Hristeva L, Booy R, Bowler I, Wilkinson AR. Prospective surveillance of neonatal meningitis. Arch Dis Child. 1993;69(1):14-8.

4. Wiswell TE, Baumgart S, Gannon CM, Spitzer AR. No lumbar puncture in the evaluation for early neonatal sepsis: will meningitis be missed? Pediatrics. 1995;95(6):803-6.

5. MacMahon P, Jewes L, de Louvois J. Routine lumbar punctures in the newborn--are they justified? Eur J Pediatr. 1990;149(11):797-9.

6. Visser VE, Hall RT. Lumbar puncture in the evaluation of suspected neonatal sepsis. J Pediatr. 1980;96(6):1063-7.

7. Cohen-Wolkowiez M, Smith PB, Mangum B, Steinbach WJ, Alexander BD, Cotten CM, et al. Neonatal Candida meningitis: significance of cerebrospinal fluid parameters and blood cultures. J Perinatol. 2007;27(2):97-100. doi:10.1038/sj.jp.7211628

8. Ajayi OA, Mokuolu OA. Evaluation of neonates with risk for infection/ suspected sepsis: is routine lumbar puncture necessary in the first 72 hours of life? Trop Med Int Health. 1997;2(3):284-8. doi: 10.1046/ j.1365-3156.1997.d01-270.x
9. Schwersenski J, McIntyre L, Bauer CR. Lumbar puncture frequency and cerebrospinal fluid analysis in the neonate. Am J Dis Child. 1991;145(1):54-8.

10. Kumar P, Sarkar S, Narang A. Role of routine lumbar puncture in neonatal sepsis. J Paediatr Child Health. 1995;31(1):8-10. doi: 10.1111/ j.1440-1754.1995.tb02902.x.

11. Hristeva L, Bowler I, Booy R, King A, Wilkinson AR. Value of cerebrospinal fluid examination in the diagnosis of meningitis in the newborn. Arch Dis Child. 1993;69(5 Spec No):514-7.

12. Tunkel AR, Hartman BJ, Kaplan SL, Kaufman BA, Roos KL, Scheld WM, et al. Practice guidelines for the management of bacterial meningitis. Clin Infect Dis. 2004;39(9):1267-84.

13. Malbon K, Mohan R, Nicholl R. Should a neonate with possible late onset infection always have a lumbar puncture? Arch Dis Child. 2006;91(1):75-6.

14. Joshi P, Barr P. The use of lumbar puncture and laboratory tests for sepsis by Australian neonatologists. J Paediatr Child Health. 1998;34(1):74-8. doi: 10.1046/j.1440-1754.1998.00158.x

15. Zea-Vera A, Turin CG, Ochoa TJ. [Unifying criteria for late neonatal sepsis: proposal for an algorithm of diagnostic surveillance]. Rev Peru Med Exp Salud Publica. 2014;31(2):358-63.

16. Shiva F, Mosaffa N, Khabbaz R, Padyab M. Lumbar puncture in neonates under and over 72 hours of age. J Coll Physicians Surg. 2006;16(8):525-8.

17. Johnson CE, Whitwell JK, Pethe K, Saxena K, Super DM, et al. Term newborns who are at risk for sepsis: are lumbar punctures necessary? Pediatrics. 1997;99(4):E10.

18. Baker MD, Bell LM. Unpredictability of serious bacterial illness in febrile infants from birth to 1 month of age. Arch Pediatr Adolesc Med 1999; 153(5):508-11.

19. Speidel BD. Adverse effects of routine procedures on preterm infants. Lancet. 1978;1(8069):864-6. doi: http://dx.doi.org/10.1016/S01406736(78)90204-0.

20. de Man P, Verhoeven BA, Verbrugh $\mathrm{HA}$, Vos MC, van den Anker JN. An antibiotic policy to prevent emergence of resistant bacilli. Lancet. 2000;355(9208):973-8. doi: http://dx.doi.org/10.1016/S01406736(00)90015-1.

21. Cotten CM, McDonald S, Stoll B, Goldberg RN, Poole K, Benjamin DK, et al. The association of thirdgeneration cephalosporin use and invasive candidiasis in extremely low birth-weight infants. Pediatrics. 2006;118(2):717-22.

22. Clark RH, Bloom BT, Spitzer AR, Gerstmann DR. Empiric use of ampicillin and cefotaxime, compared with ampicillin and gentamicin, for neonates at risk for sepsis is associated with an increased risk of neonatal death. Pediatrics. 2006;117(1):67-74.

Correspondencia: Theresa J. Ochoa

Dirección: Av. Honorio Delgado 430, San

Martin de Porras, Lima 33, Perú

Teléfono: (+511) 319-0000 ext 2715; Fax 51-1-482-3404

Correo electrónico: theresa.j.ochoa@uth.tmc.edu 